\title{
Specialty substance use disorder services following brief alcohol intervention: a meta-analysis of randomized controlled trials
}

\author{
Joseph E. Glass', Ashley M. Hamilton², Byron J. Powell ${ }^{3}$, Brian E. Perron ${ }^{4}$, Randall T. Brown ${ }^{5}$ \\ \& Mark A. Ilgen ${ }^{6}$
}

School of Social Work, University of Wisconsin-Madison, Madison, WI, USA, 'Chrysalis, Inc., Madison, WI, USA, ${ }^{2}$ Department of Psychiatry, Perelman School of Medicine, University of Pennsylvania, Philadelphia, PA, USA, ${ }^{3}$ School of Social Work, University of Michigan, Ann Arbor, MI, USA, Department of Family Medicine, School of Medicine and Public Health, University of Wisconsin-Madison, Madison, WI, USA ${ }^{5}$ and VA Center for Clinical Management Research (CCMR), VA Ann Arbor Healthcare System and the Department of Psychiatry, University of Michigan Medical School, Ann Arbor, MI, USA 6

\begin{abstract}
Background and aims Brief alcohol interventions in medical settings are efficacious in improving self-reported alcohol consumption among those with low-severity alcohol problems. Screening, Brief Intervention and Referral to Treatment initiatives presume that brief interventions are efficacious in linking patients to higher levels of care, but pertinent evidence has not been evaluated. We estimated main and subgroup effects of brief alcohol interventions, regardless of their inclusion of a referral-specific component, in increasing the utilization of alcohol-related care. Methods A systematic review of English language papers published in electronic databases to 2013. We included randomized controlled trials (RCTs) of brief alcohol interventions in general health-care settings with adult and adolescent samples. We excluded studies that lacked alcohol services utilization data. Extractions of study characteristics and outcomes were standardized and conducted independently. The primary outcome was post-treatment alcohol services utilization assessed by self-report or administrative data, which we compared across intervention and control groups. Results Thirteen RCTs met inclusion criteria and nine were meta-analyzed ( $n=993$ and $n=937$ intervention and control group participants, respectively). In our main analyses the pooled risk ratio $(\mathrm{RR})$ was $=1.08,95 \%$ confidence interval $(\mathrm{CI})=0.92-1.28$. Five studies compared referral-specific interventions with a control condition without such interventions (pooled RR $=1.08,95 \%$ $\mathrm{CI}=0.81-1.43$ ). Other subgroup analyses of studies with common characteristics (e.g. age, setting, severity, risk of bias) yielded non-statistically significant results. Conclusions There is a lack of evidence that brief alcohol interventions have any efficacy for increasing the receipt of alcohol-related services.
\end{abstract}

Keywords Alcohol, brief intervention, meta-analysis, referral to treatment, treatment initiation, treatment utilization.

Correspondence to: Joseph E. Glass, School of Social Work, University of Wisconsin-Madison, 1350 University Avenue, Madison, WI 53706, USA. E-mail: jglass2@wisc.edu

Submitted 28 November 2014; initial review completed 10 February 2015; final version accepted 10 April 2015

\section{INTRODUCTION}

Unhealthy alcohol use includes a spectrum of alcohol use, ranging from risky drinking to a clinically diagnosed alcohol use disorder [1]. Unhealthy alcohol use is the third leading cause of death in the United States [2,3], and is estimated to cost the United States over $\$ 230$ billion annually [4]. Approximately 17.6 million adults in the United States meet criteria for a past-year alcohol use disorder, but just $6 \%$ of these individuals receive treatment and only $11 \%$ report that they need or want help for their drinking [5,6]. Although very few individuals receive treatment for their alcohol problems [7], various forms of alcohol treatment are cost-effective and improve clinical outcomes [8-15].

Although few people attend medical care to address their drinking, health-care visits present an opportunity to identify alcohol problems through universal screenings and to provide brief advice or motivational interventions to encourage individuals to reduce their drinking [16-18]. Alcohol screening and brief intervention (SBI) in medical settings is efficacious for those with mild to moderate 
alcohol problems [19], including those who meet the National Institute on Alcohol Abuse and Alcoholism's definition of at-risk drinking or DSM-IV criteria for alcohol abuse [20,21], but it may not be either as applicable or as effective in those with more severe patterns of use. SBI guidelines recommend that individuals with severe forms of unhealthy alcohol use such as DSM-IV alcohol dependence [20] be referred to more intensive services, such as treatments with addiction specialists $[18,21,22]$. To address the full spectrum of unhealthy alcohol use, including those with severe problems, Screening, Brief Intervention and Referral to Treatment (SBIRT) programs call attention to efforts to refer individuals to treatment [18]. For instance, SBIRT emphasizes the coordination between community services systems (e.g. general health-care and specialty addiction treatment agencies) to improve the quality and success of referrals [18]. Theoretically, SBIRT extends SBI to those with more severe conditions, with services matched to the level of need of all individuals with unhealthy alcohol use [18].

Although several systematic or meta-analytical reviews have demonstrated the effectiveness of brief alcohol interventions in reducing alcohol consumption [23-25], the ability of SBI or SBIRT programs to increase the utilization of alcohol-related care needs further investigation. Surprisingly, reviews of SBIRT have not attempted to evaluate the referral to treatment components of these programs [26,27], or have found insufficient evidence to do so $[28,29]$. For instance, one systematic review sought to examine the efficacy of SBIRT in adolescents, but it identified no randomized controlled trials (RCTs) that either evaluated referral to treatment or reported the percentage of participants eligible for referral based on screening results [28]. Existing reviews of SBI have also not provided adequate data to inform the referral to treatment component of SBIRT. A systematic review in 2010 of RCTs of SBI in primary care settings that included drinkers with alcohol dependence found no studies that examined linkage to alcohol treatment as a study outcome [30]. Two other systematic reviews (including one meta-analysis) evaluated the effect of brief interventions on subsequent health-care utilization (i.e. in-patient, out-patient and emergency care), but neither specifically examined utilization of specialty alcohol treatments [31,32]. Overall, reviews have produced insufficient evidence to evaluate whether or not brief alcohol interventions actually increase subsequent treatment utilization.

Given the current state of the literature, we reviewed and meta-analyzed RCTs systematically to evaluate the extent to which brief alcohol interventions in medical settings are effective in linking people to alcohol-focused services. Our primary analyses compared the outcome of posttreatment alcohol services utilization across intervention and control groups of RCTs. The effect of SBI on the utilization of alcohol-related care could depend upon intervention characteristics such as intervention intensity (e.g. number of sessions) [33] and the presence of active efforts to refer individuals to alcohol-related care (e.g. addressing concerns about obtaining addiction treatment) [34], so we conducted subgroup analyses to account for clinical heterogeneity [35]. The evidence for SBI has been evaluated separately in several reviews based on other study characteristics, such as age (adolescent versus adult) [28,36], clinical setting [37,38] and alcohol use severity [30], which we also considered in subgroup analyses. Lastly, our secondary goal was to evaluate whether or not alcohol-related outcomes improved among those who were referred to higher levels of care, which is the purpose of referring people to treatment [34]. To accomplish this goal, we conducted a qualitative review of results from the RCTs of brief alcohol interventions that attempted to evaluate the association between alcohol treatment utilization and clinical outcomes.

\section{METHOD}

This review followed guidelines outlined in the Preferred Reporting Items for Systematic Reviews and Meta-analysis (PRISMA) statement [39].

\section{Inclusion criteria}

We included studies meeting the following criteria: (1) RCT, (2) intervened with unhealthy alcohol use in medical settings, (3) study sample was not seeking alcohol treatment at the time of recruitment, (4) linkage to alcohol-related services, such as specialty addiction treatment or mutual help programs, was assessed as an intervention outcome or as a mediator of intervention outcomes, and (5) published in the English language. SBIRT programs vary in the specific referral processes used to link clients to treatment [18]; hence, we did not restrict studies to any particular treatment or referral modality. We did not limit our inclusion of studies to any specific country.

\section{Exclusion criteria}

Because our focus was on alcohol-related brief intervention approaches, we excluded studies involving participants with drug but not alcohol use. To maximize generalizability, we excluded studies in which the outcome consisted of attendance at treatment sessions that were delivered by clinical research interventionists as part of the research study [40,41]. It was not an objective of our study to evaluate the integration of addiction treatment into general health-care settings $[42,43]$. We excluded these studies, which may have evaluated the receipt of alcohol-related care within the same setting, because their results would 
probably not generalize to SBI or SBIRT programs. Integrated treatments use different transition practices (e.g. consults to providers in the same clinic) and require different organizational resources (e.g. embedded addiction specialists), yet they generally provide less intensive services than specialty treatment programs targeted by referrals [34].

\section{Data sources and searches}

We conducted an extensive database search, expert query and hand search to identify papers. The database search, which was conducted with assistance from a reference librarian, identified peer-reviewed studies published in the English language until 26 July 2013 in MEDLINE, PsycINFO and CINAHL Plus (see Supporting information, Table S1 for search keywords). A hand search, which targeted peer-reviewed papers and grey literature, was conducted by examining references of the included studies, SBIRT bibliographies and several relevant review papers [18,26-28,31,32,44,45]. Authors of the identified studies were e-mailed, requesting that they provide knowledge of existing studies related to the review.

\section{Study selection}

Abstracts were screened and discarded if studies clearly did not meet the inclusion/exclusion criteria. Remaining papers were retrieved for the full-text review. The first author read and evaluated each paper against the inclusion/exclusion criteria, seeking assistance from other authors when necessary.

\section{Data extraction and quality assessment}

Two authors independently reviewed papers identified by the literature search to extract data regarding outcomes, study characteristics and risk of bias. Data extraction was standardized with forms and meetings were held to review study data and to identify and resolve discrepancies.

\section{Primary outcome measure}

We extracted raw counts of alcohol treatment utilization for the treatment and control groups or derived the raw counts if only percentages were reported. We also recorded whether a statistically significant difference was found when comparing post-intervention drinking-related outcomes and alcohol treatment utilization between intervention and control groups. Study authors were emailed to request these data if not provided in the publication.

\section{Study characteristics}

We grouped studies by the age of the sample (adolescent versus adult). We also extracted data on the health-care setting of the intervention (medical in-patient units, general health-care settings, emergency departments) [31]. We classified intervention intensity as low for interventions with no in-person contact, medium for studies with a single session intervention and high for multiple-session interventions. The severity of alcohol use in the study samples was classified as high for strictly alcoholdependent samples, alcohol detoxification samples or samples recruited for having severe alcohol-induced medical problems; low for samples that excluded dependent drinkers; and mixed for samples with a broad range of alcohol use (e.g. included both risky/problem drinking and alcohol dependence, included dependent and non-dependent drinkers but excluded heavy drinkers). We also recorded all information about referral-specific interventions that were included in the intervention and control groups (e.g. providing lists of treatment agencies to the participants) and noted when referral-specific interventions were isolated to the treatment group.

\section{Data synthesis and analysis}

Meta-analysis was performed with the metan package in Stata version 13 [46]. We calculated risk ratios (RR) and 95\% confidence intervals (CI) in models with random effects and used a forest plot to visualize the findings. Heterogeneity among studies was assessed with the $I^{2}$ statistic, which describes the proportion of variation across studies due to heterogeneity versus chance [47]. We performed subgroup analysis of the studies based on study characteristics (i.e. age, setting, intervention intensity and population severity) and conducted a sensitivity analysis by excluding studies with a risk of bias in more than two areas.

\section{Risk of bias}

Study-level risk of bias was ascertained with the following characteristics: randomization concealment, proportion of participants lost to follow-up [48], standardization of intervention delivery (e.g. trained interventionists, followed treatment manuals) and presence of an intent to treat analysis [48]. Outcome-level risk of bias focused on the validity of the treatment utilization analyses, including the measurement properties of the instruments [49,50] and blinding of outcome assessment [51]. Risk of bias was assessed for the purpose of sensitivity analysis (see Meta-analysis).

\section{Publication bias}

We checked for systematic bias in reporting [52] using the metafunnel and metabias commands in Stata. We constructed a funnel plot of each trial's effect size against its 
standard error then used the Harbord test for binary outcome data [53] to examine the association between study effect sizes and sample sizes.

\section{RESULTS}

\section{Study characteristics}

The literature search yielded 13 independent RCTs of brief alcohol interventions that evaluated post-intervention alcohol treatment utilization (see Fig. 1). Supporting information, Table 2 provides reasons for exclusion of several studies that met inclusion/exclusion criteria most closely. Interventions were delivered in medical in-patient units [33,54], general health-care settings [55-57] and emergency departments (see Table 1) [58-65]. Four RCTs were conducted outside the United States, including France, Germany, Poland and Australia [55,57,59,60]. The majority of interventions involved brief advice or a motivational interview [33,54,58,59,61,62,64-66]; several offered additional counseling or booster intervention sessions $[55,57,61,66]$ and one intervention had no in-person contact and simply mailed a letter to participants requesting they make an appointment with a specialist [60].

\section{Referral-specific components of the interventions}

Although all studies conducted efforts that could potentially inspire help-seeking (e.g. motivational sessions), five of the 13 studies did not articulate any referral-specific processes in the intervention group [33,55-58] and the remaining eight studies described a referral-specific intervention. All these eight studies provided information about alcohol treatment options in the community [54,59-65], and three of them described more active efforts to encourage help-seeking. Active efforts to encourage help-seeking included one study that had an intervention session devoted to discussing treatment options [54], another study that included a booster session where previously supplied treatment referral materials were reviewed [61] and another study that mailed a letter to patient homes to encourage them to make an appointment with a specific treatment center [60].

In total, six of the eight studies with referral-specific efforts isolated these referral-specific efforts to the intervention group [54,59-63]. The other two studies provided only information about treatment options as the referralspecific effort, but provided the same information to the control group [64,65]. A more detailed description of the intervention and control groups of the studies is included in Supporting information, Table 3.

\section{Measurement of alcohol-related care}

In 11 of 13 studies, the presence of treatment utilization was defined as receiving one or more sessions of specialty addiction treatment [33,54,55,58-65], whereas the remaining two studies analyzed counts of specialty addiction treatment visits $[56,57]$. Four studies used treatment agency or state administrative data to assess treatment utilization $[54,56,60,61]$ and the rest used self-report. Studyspecific measures of self-reported treatment utilization were common [55,57,59,62-65]; just two studies assessed self-reports of alcohol treatment utilization with validated instruments $[33,58]$. Follow-up periods ranged from 3 to 18 months, except for one study [57] that had a 10-year follow-up.

\section{Utilization of alcohol-related care}

Just one study found a significant difference between treatment and control groups in the utilization of alcohol treatment (see Table 2) [60]. Studies conducted in in-patient settings and/or with high-severity samples tended to have the highest rates of post-intervention alcohol treatment utilization $(18.9-56.1 \%$ in the intervention groups obtained alcohol treatment) [33,54,55]. In comparison,
Figure I Flow diagram depicting the process for identifying studies. All papers identified by the hand search or author query that met criteria for inclusion were also identified in the database search. No grey literature that met our inclusion/exclusion criteria was identified

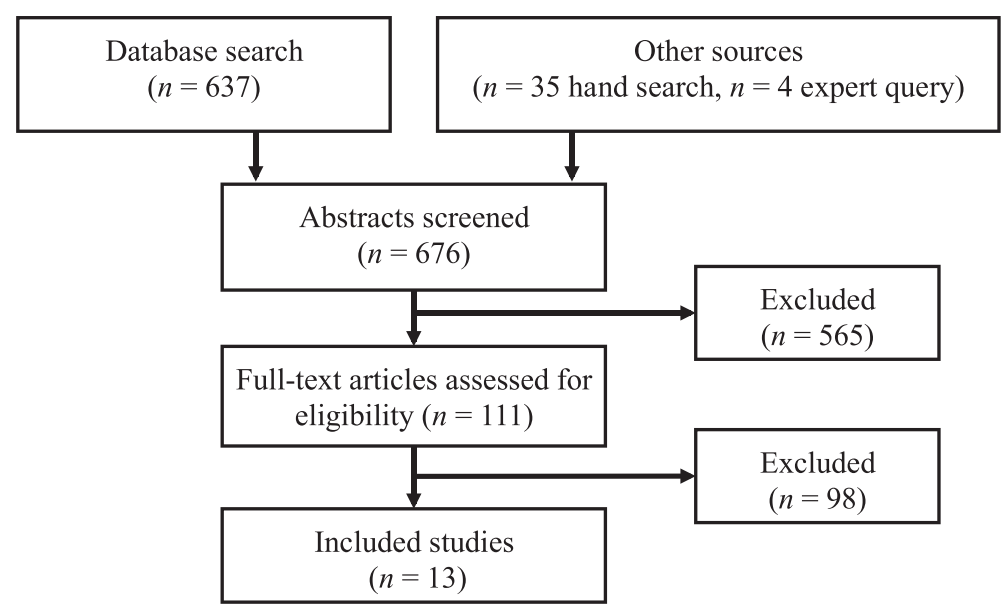




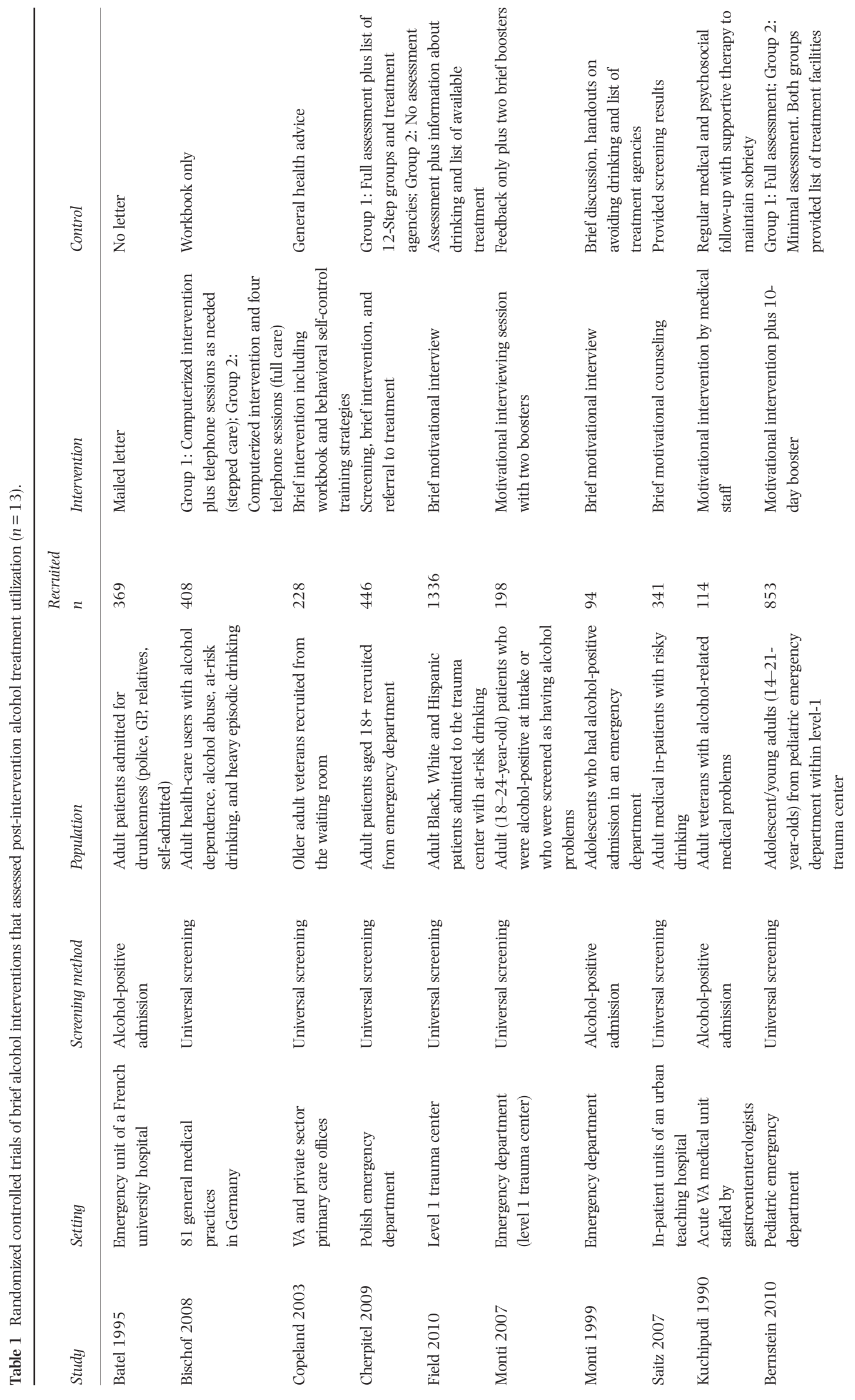




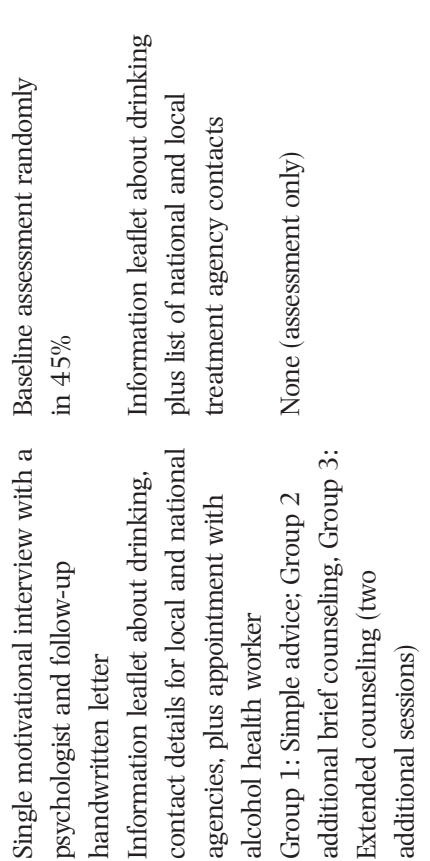

ชู के in

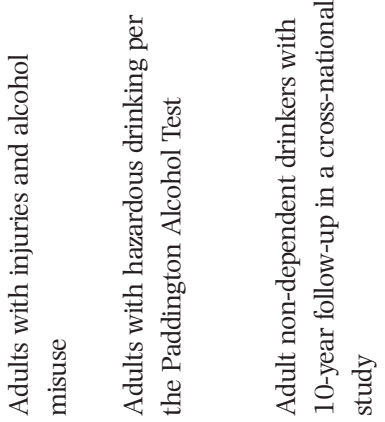

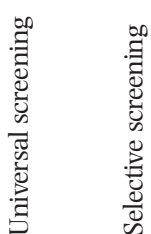

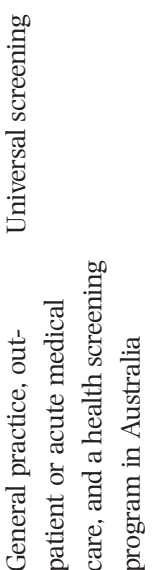

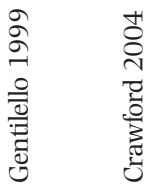

studies conducted in emergency departments and/or with mixed severity samples had a lower range of post-intervention alcohol treatment utilization (1.9-32.8\% in the intervention groups obtained alcohol treatment) [59-63,66].

\section{Alcohol-related outcomes}

Nine of 13 studies reported improvements in one or more drinking-related outcomes due to brief intervention at one or more time-points [55,57-59,61-65], and two studies did not examine drinking-related outcomes [56,60]. Just two studies considered the association between alcohol treatment utilization and alcohol-related outcomes. One found that SBI was associated with reductions in drinking and driving, moving violations, alcohol-related injuries and alcohol-related problems, but stated that the small sample size $(n=94)$ precluded a formal statistical analysis to evaluate whether post-intervention alcohol treatment utilization mediated the association between brief intervention and treatment outcomes [65]. A second study also did not conduct a formal mediation analysis, but stated that the effects of SBI on alcohol consumption was not due to the receipt of specialty alcohol treatment, because postintervention treatment utilization rates were similarly low in the intervention and control groups (4.7 versus $4.8 \%$, respectively, received formal treatment and 15.6 versus $13.7 \%$, respectively, attended self-help groups) [62].

\section{Risk of bias}

Supporting information, Table 4 contains results of the risk of bias assessment. Although all studies discussed aspects of randomization, only one reported off-site assignment [33] or sequential assignment of sealed opaque cards to ensure concealment of randomization. The study that had a high risk of bias in four of six domains was also the only study that found significant effects on treatment utilization [60].

\section{Publication bias}

The funnel plot and Harbord tests did not produce evidence of publication bias.

\section{Meta-analysis}

Sufficient data were obtained from 10 studies to metaanalyze the association between receipt of brief intervention and subsequent alcohol treatment initiation (see Table 2). We focus our meta-analyses on nine of 10 studies that had available data, excluding the study with a risk of bias in four of six domains [60]. In these nine studies, there were $n=993$ and $n=937$ intervention and control group participants, respectively. Receipt of brief intervention was not associated significantly with subsequent alcohol 


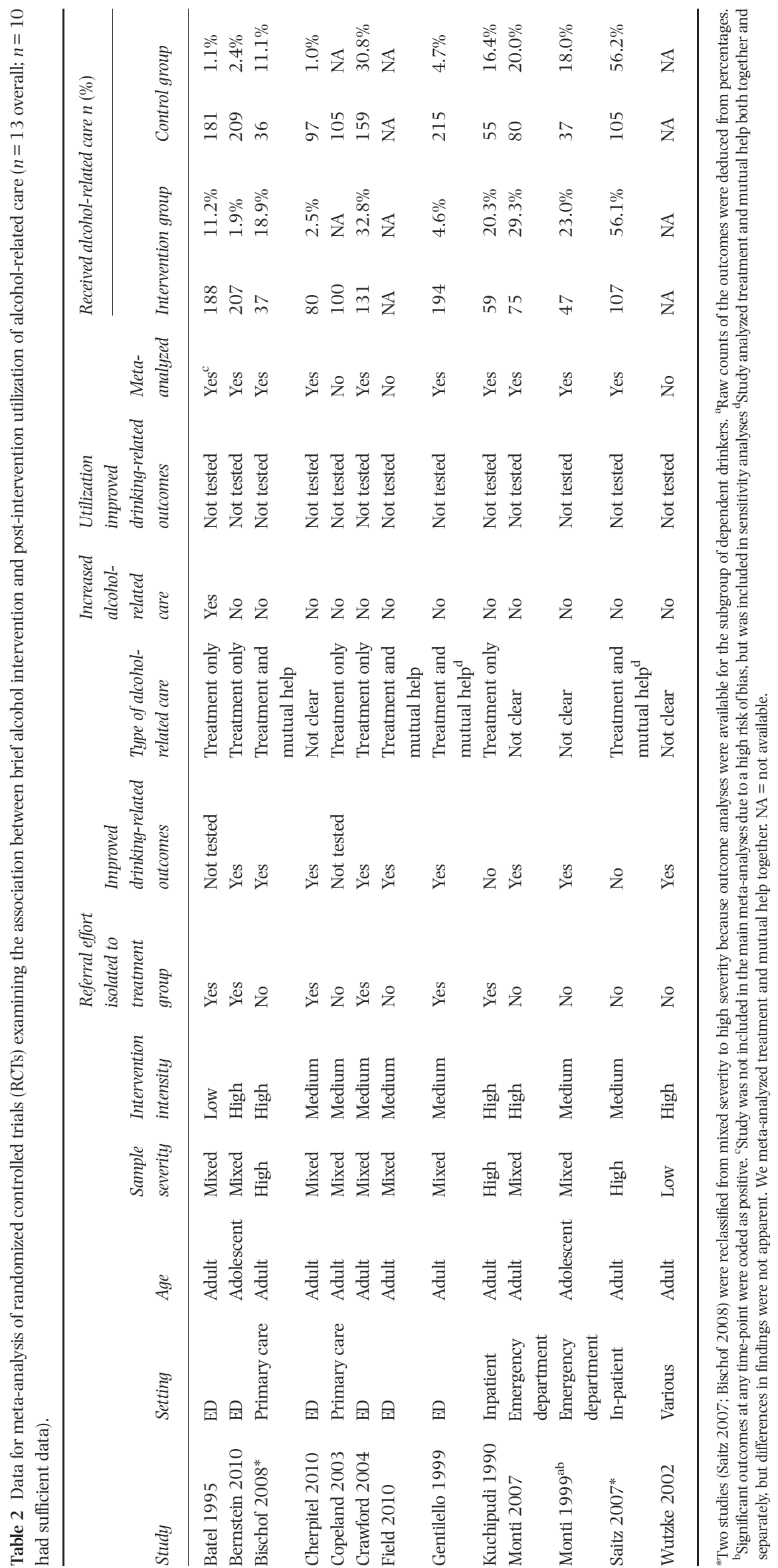


treatment initiation. The random-effects pooled risk ratio was $\mathrm{RR}=1.08$, 95\% CI $=0.92-1.28$ (see Fig. 2). The $I^{2}$ statistic was $0 \%$, indicating no evidence of study heterogeneity. Pooled results of studies that isolated referral to treatment to one study arm $(n=5)$ did not achieve statistical significance $(\mathrm{RR}=1.08,95 \% \mathrm{CI}=0.81-1.43)$. Other subgroup analyses, which pooled results of multiple studies with similar characteristics (i.e. by age, setting, severity and treatment intensity), also yielded non-statistically significant risk ratios (e.g. $\mathrm{RR}=1.08$, 95\% CI $=0.91-1.29$ for adult studies, $\mathrm{RR}=1.09$, 95\% $\mathrm{CI}=0.54-2.21$ for adolescent studies; $\mathrm{RR}=1.04$, 95\% $\mathrm{CI}=0.83-1.30)$ for high-severity studies; other subgroupspecific risk ratios not shown). Excluding studies that had a risk of bias in more than two areas of risk did not alter the results. Moreover, we note that including the study with a risk of bias in four of six domains, which was the only study that achieved statistical significance, also did not alter the results (e.g. pooled results for all 10 studies was $\mathrm{RR}=1.22$, 95\% CI $=0.94-1.58$ ). There were insufficient data (i.e. no identified studies) to meta-analyze whether or not alcohol-related outcomes improved as a result of referral to treatment.

\section{DISCUSSION}

The primary purpose of this study was to estimate the efficacy of brief alcohol interventions in linking people to higher levels of alcohol-related care. Based on a synthesis of 13 RCTs that met inclusionary requirements, which included pooled and subgroup-specific meta-analysis of nine RCTs, we found no evidence that brief alcohol interventions were effective in increasing the utilization of alcohol-related care. This lack of evidence calls into question the assumption that referral to treatment as part of SBI or SBIRT effectively links patients to higher levels of care for their alcohol problems.

Samples with higher alcohol severity and/or those recruited from more severe settings (e.g. in-patient medical settings) $[33,54,55]$ tended have higher rates of service utilization than samples with lower alcohol severity and/or those recruited from general health-care settings [59-63,66]. It seems logical that baseline severity would be an important moderator of effects of referral to treatment interventions. For instance, it is likely that referral to a higher level of care would not be indicated for the majority of participants in studies that included hazardous and harmful drinkers but excluded dependent drinkers [57]. Given that SBI targets individuals with a broad range of alcohol severity, severity-stratified analyses and subgroup analyses [33,55] may be the most valid approach to evaluating the efficacy of referral to treatment. None the less, our subgroup-specific meta-analysis found that regardless of sample severity, brief interventions were not efficacious in increasing alcohol treatment utilization. In the subgroup of studies with samples that were deemed as high severity $[33,54,55]$, only one of these studies isolated an active referral effort to the treatment group [54]. It is possible that, with more studies and greater power, we could have conducted a more thorough analysis of subgroup effects.

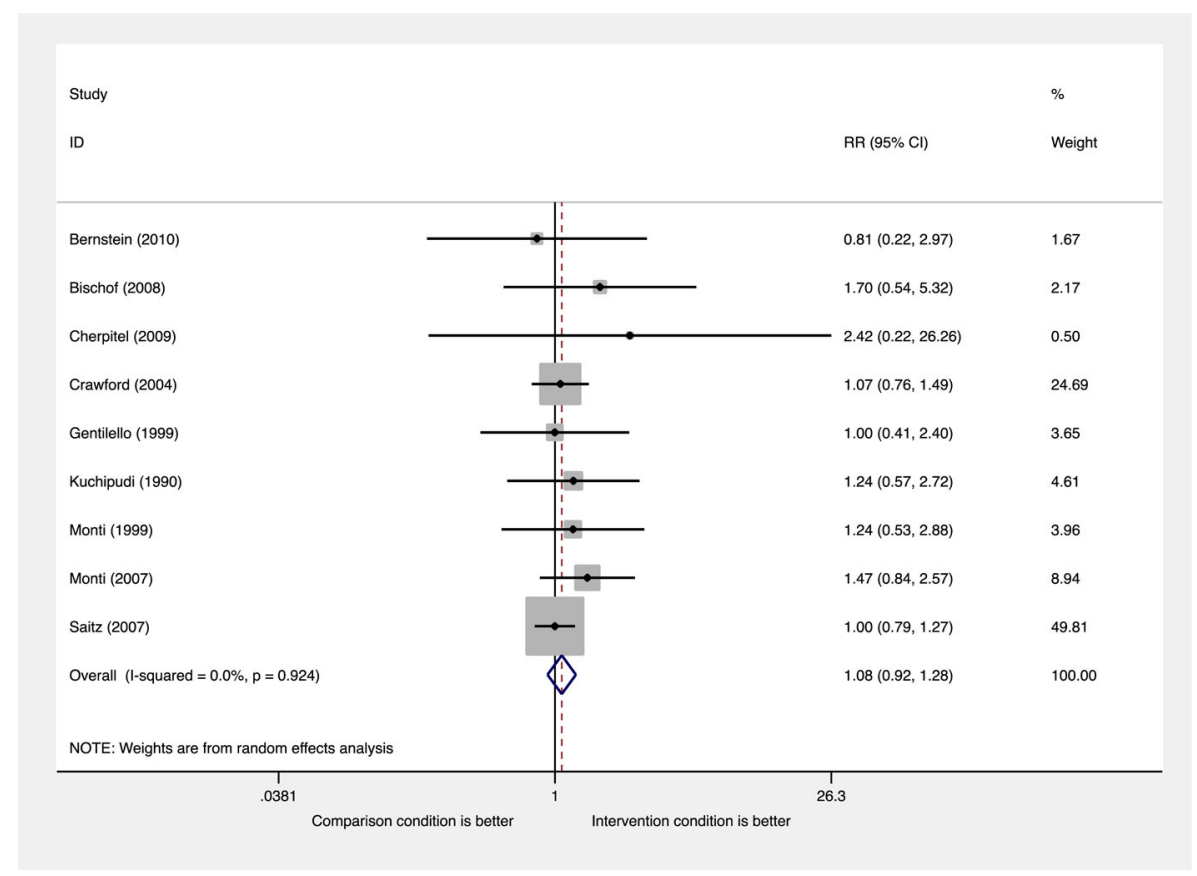

Figure 2 The forest plot contains risk ratios and confidence intervals for each study in the meta-analysis and a pooled risk ratio and confidence interval (depicted by the diamond) calculated with random effects. The areas of the squares are proportional to study weights in the meta-analysis 
Although rates of specialty treatment utilization following brief intervention ranged from 2 to $56 \%$, no studies analyzed an association between treatment utilization and clinical outcomes. Therefore, there was a lack of data to evaluate whether or not referral to and receipt of specialty alcohol treatment improved clinical outcomes among brief alcohol intervention recipients. Importantly, the diverse study characteristics with regard to sample severity and intervention content suggests that there is a need to develop and evaluate methods systematically for referring people with severe unhealthy alcohol use to higher levels of care. Particularly in general medical settings, individuals with less severe forms of unhealthy alcohol use are more common than those with more severe problems, including those with clinical alcohol use disorders. It is likely that clinicians who deliver brief interventions spend the majority of their time working with lower-severity individuals. Much has been written about how to provide brief interventions to lower-severity drinkers [21,67], but less guidance exists to inform the development of effective intervention materials focused on referring patients to specialty alcohol treatments. Indeed, many of the identified studies were limited in their description of referral processes [56-58,64,65], or simply provided lists of local alcohol treatment agencies to participants [59,62]. Qualitative data from one SBIRT study found that clinicians felt that they should not provide referrals to patients unless they were specifically requested due to the stigma associated with alcohol treatment [68]. Thus, it may be important to consider how provider factors, such as the perceived stigma of unhealthy alcohol use and its treatment [69], hinder brief intervention and treatment referral practices within medical settings [70,71]. While health-care professional groups have mandated or have discussed mandating SBIRT, it is important to have realistic expectations about the potential impact of these approaches within health systems.

In the identified studies, the majority of participants who received brief interventions did not subsequently attend alcohol treatment. As has been noted by prior reviews, this highlights the fact that it is difficult to encourage people to utilize alcohol treatment [30]. For instance, some have discussed the fact that specialty addictions treatment is not appealing to many individuals [72]. Even higher-intensity brief interventions may be insufficient for laying the foundation for subsequent treatment initiation when discussions do not address barriers to attending treatment, concerns about treatment efficacy and/or provide education about behavioral and pharmacological treatments offered in these settings [73]. Conceptual frameworks have characterized brief alcohol intervention and referral as a low-intensity approach to referring individuals to specialty care, and suggest that more intensive efforts (e.g. telephone monitoring, continued contact, case management) may be necessary to provide effective linkages [34]. In addition to improving interventions that link people in medical settings to alcohol treatment, the continued development and implementation of treatments for alcohol use disorders into general health-care settings, including alcohol pharmacotherapy [74], primary caremental health integration [75] and chronic alcohol care management [76] will remain critical in order to meet the needs of individuals in medical settings with severe forms of unhealthy alcohol use. In addition, it would be worthwhile to evaluate whether specific subgroups of individuals benefit from new and existing efforts to increase the receipt of alcohol-related care. For instance, although their main effects were not statistically significant [33], one study found that brief intervention increased treatment utilization among women and adults below age 44 years [77].

\section{Limitations}

Our exclusion of non-English language papers may have resulted in us missing some important studies. Many RCTs of brief interventions have been conducted, but most do not assess treatment utilization and many exclude participants who would benefit from specialty care (e.g. those with DSM-IV dependence) [30]. Although there was no evidence for publication bias, our hand search to identify grey literature could have missed unpublished reports. However, RCTs with positive results tend to be published [78], thus it is unlikely that our findings would be substantively altered by unidentified unpublished research. There was significant heterogeneity across studies on important factors (e.g. age, treatment intensity), although the metaanalytical results did not change when analyzing subgroups of studies. Although the heterogeneity statistic calculated by the meta-analysis was acceptable, the estimated relative risk ratios should not be generalized to all settings and populations. Several of the included studies were limited in their descriptions of referral-specific components of the interventions provided in the treatment and control groups, which highlights a need for better reporting in clinical trials. While treatment utilization is a low-frequency outcome, only one of the included studies described a power analysis to detect this effect [33]. The assessment of alcohol-related care varied across studies, and in some studies was not described sufficiently to determine the types of care that were assessed.

\section{Implications}

Connecting individuals to higher levels of care is a theoretically important part of SBIRT programs to provide effective forms of treatment to people with severe alcohol problems [18,21]. Despite the widespread support for 
SBIRT implementation as a public health program to address all forms of unhealthy alcohol use, there is a lack of evidence from existing studies of brief alcohol interventions to support the assumption that SBIRT, as currently implemented, is efficacious in linking individuals to higher levels of alcohol-related care. Given the importance of this aspect of SBIRT, one might question whether or not SBIRT currently addresses the full spectrum of unhealthy alcohol use. However, most existing RCTs have not been designed with the evaluation of the utilization of alcohol-related care as their primary focus, which suggests a need for more clinical trials with a primary focus on referral to treatment. Importantly, SBIRT has not been tested with more intensive linkage programs [79], which may be more effective with more severe patients. Future clinical trials should evaluate referral to treatment as a primary outcome, sufficiently explicate and track referral processes and consider the alcohol severity of the samples that are evaluated.

\section{Declaration of interests}

None.

\section{Acknowledgements}

We are grateful to Wendy F. Auslander $\mathrm{PhD}$, Washington University in St Louis, and Sarah C. Narendorf PhD, University of Houston, for their feedback on this work. We would also like to recognize the anonymous reviewers of Addiction for their helpful feedback.

\section{References}

1. Saitz R. Clinical practice. Unhealthy alcohol use. N Engl J Med 2005; 352: 596-607.

2. Mokdad A. H., Marks J. S., Stroup D. F., Gerberding J. L. Actual causes of death in the United States, 2000. JAMA 2004; 291 : $1238-45$

3. Center for Disease Control and Prevention. Alcohol and public health: data, trends, and maps [internet]. 2014 (cited 3 July 2014). Available at: http://www.cdc.gov/alcohol/quickstats/ binge_drinking.htm (Archived by WebCite ${ }^{\circledR}$ at: http://www. webcitation.org/6YWH25GOV) (accessed 13 May 2015).

4. Rehm J., Mathers C., Popova S., Thavorncharoensap M., Teerawattananon Y., Patra J. Global burden of disease and injury and economic cost attributable to alcohol use and alcohol-use disorders. Lancet 2009; 373: 2223-33.

5. Grant B. F., Stinson F. S., Dawson D. A., Chou S. P., Dufour M. C., Compton W. et al. Prevalence and co-occurrence of substance use disorders and independent mood and anxiety disorders: results from the National Epidemiologic Survey on Alcohol and Related Conditions. Arch Gen Psychiatry 2004; 61: 807-16.

6. Edlund M. J., Unutzer J., Curran G. M. Perceived need for alcohol, drug, and mental health treatment. Soc Psychiatry Psychiatr Epidemiol 2006; 41: 480-7.

7. Glass J. E., Perron B. E., Ilgen M. A., Chermack S. T., Ratliff S., Zivin K. Prevalence and correlates of specialty substance use disorder treatment for Department of Veterans Affairs
Healthcare System patients with high alcohol consumption. Drug Alcohol Depend 2010; 112: 150-5.

8. Dawson D. A., Grant B. F., Stinson F. S., Chou P. S., Huang B., Ruan W. J. Recovery from DSM-IV alcohol dependence: United States, 2001-2002. Addiction 2005; 100: 281-92.

9. Dawson D. A., Grant B. F., Stinson F. S., Chou P. S. Estimating the effect of help-seeking on achieving recovery from alcohol dependence. Addiction 2006; 101: 824-34.

10. Ettner S. L., Huang D., Evans E., Ash D. R., Hardy M., Jourabchi M. et al. Benefit-cost in the California treatment outcome project: does substance abuse treatment 'pay for itself'? Health Serv Res 2006; 41: 192-213.

11. Finney J. W., Wilbourne P. L., Moos R. H. Psychosocial Treatments for Substance Use Disorders. A Guide to Treatments That Work. New York: Oxford University Press; 2007, pp. 179-202.

12. Fleming M. F., Mundt M. P., French M. T., Manwell L. B., Stauffacher E. A., Barry K. L. Benefit-cost analysis of brief physician advice with problem drinkers in primary care settings. Med Care 2000; 38: 7-18.

13. Miller W. R., Wilbourne P. L. Mesa Grande: a methodological analysis of clinical trials of treatments for alcohol use disorders. Addiction 2002; 97: 265-77.

14. Moos R. H., Moos B. S. Rates and predictors of relapse after natural and treated remission from alcohol use disorders. Addiction 2006; 101: 212-22.

15. Zarkin G. A., Bray J. W., Aldridge A., Mills M., Cisler R. A., Couper D. et al. The effect of alcohol treatment on social costs of alcohol dependence: results from the COMBINE study. Med Care 2010; 48: 396-401.

16. Babor T. F., Ritson E. B., Hodgson R. J. Alcohol-related problems in the primary health care setting: a review of early intervention strategies. Br J Addict 1986; 81: 23-46.

17. Chafetz M. E., Blane H. T., Abram H. S., Golner J., Lacy E., McCourt W. et al. Establishing treatment relations with alcoholics. J Nerv Ment Dis 1962; 134: 395-409.

18. Babor T. F., McRee B. G., Kassebaum P. A., Grimaldi P. L., Ahmed K., Bray J. Screening, Brief Intervention, and Referral to Treatment (SBIRT): toward a public health approach to the management of substance abuse. Subst Abuse 2007; 28: 7-30.

19. Preventive U. S. Services Task Force. Screening and behavioral counseling interventions in primary care to reduce alcohol misuse: recommendation statement. Ann Intern Med 2004; 140: 554-6.

20. American Psychiatric Association Diagnostic and Statistical Manual of Mental Disorders, 4th revised edn. Washington, DC: American Psychiatric Association; 2000 xxxvii, p., pp. 943.

21. National Institute on Alcohol Abuse and Alcoholism. Helping Patients Who Drink Too Much: A Clinician's Guide [internet]. Bethesda, MD; 2005. Available at: http://pubs.niaaa.nih.gov/ publications/Practitioner/CliniciansGuide2005/clinicians_ guide.htm (Archived by WebCite a at http://www. webcitation.org/6YWGq774x) (accessed 14 May 2015).

22. Moyer V. A., Preventive Services Task Force. Screening and behavioral counseling interventions in primary care to reduce alcohol misuse: U.S. preventive services task force recommendation statement. Ann Intern Med 2013; 159: 210-18.

23. Kaner E. F., Beyer F., Dickinson H. O., Pienaar E., Campbell F., Schlesinger C. et al. Effectiveness of brief alcohol interventions in primary care populations. Cochrane Database Syst Rev 2007; 18 CD004148. 1-93.

24. Kaner E. F., Dickinson H. O., Beyer F., Pienaar E., Schlesinger C., Campbell F. et al. The effectiveness of brief alcohol interventions in primary care settings: a systematic review. Drug Alcohol Rev 2009; 28: 301-23. 
25. Whitlock E. P., Polen M. R., Green C. A., Orleans T., Klein J. Behavioral counseling interventions in primary care to reduce risky/harmful alcohol use by adults: a summary of the evidence for the U.S. Preventive Services Task Force. Ann Intern Med 2004; 140: 557-68.

26. Whitlock E. P., Green C. A., Polen M. R., Berg A., Klein J., Siu A. et al. Behavioral Counseling Interventions in Primary Care to Reduce Risky/Harmful Alcohol Use. Rockville (MD): Agency for Healthcare Research and Quality (US); 2004

27. Ahmadi H., Green S. L. Screening, brief intervention, and referral to treatment for military spouses experiencing alcohol and substance use disorders: a literature review. J Clin Psychol Med Settings 2011; 18: 129-36.

28. Mitchell S. G., Gryczynski J., O'Grady K. E., Schwartz R. P. SBIRT for adolescent drug and alcohol use: current status and future directions. J Subst Abuse Treat 2013; 44: 463-72.

29. Young M. M., Stevens A., Galipeau J., Pirie T., Garritty C., Singh K. et al. Effectiveness of brief interventions as part of the Screening, Brief Intervention and Referral to Treatment (SBIRT) model for reducing the nonmedical use of psychoactive substances: a systematic review. Syst Rev 2014; 3: 50.

30. Saitz R. Alcohol screening and brief intervention in primary care: absence of evidence for efficacy in people with dependence or very heavy drinking. Drug Alcohol Rev 2010; 29: 631-40.

31. Bray J. W., Cowell A. J., Hinde J. M. A systematic review and meta-analysis of health care utilization outcomes in alcohol screening and brief intervention trials. Med Care 2011; 49: 287-94.

32. Mdege N. D., Fayter D., Watson J. M., Stirk L., Sowden A., Godfrey C. Interventions for reducing alcohol consumption among general hospital inpatient heavy alcohol users: a systematic review. Drug Alcohol Depend 2013; 131: 1-22.

33. Saitz R., Palfai T. P., Cheng D. M., Horton N. J., Freedner N., Dukes K. et al. Brief intervention for medical inpatients with unhealthy alcohol use: a randomized, controlled trial. Ann Intern Med 2007; 146: 167-76.

34. Cucciare M. A., Coleman E. A., Timko C. A conceptual model to facilitate transitions from primary care to specialty substance use disorder care: a review of the literature. Prim Health Care Res Dev 2014; 12: 1-14.

35. Higgins J., Green S., editors. Cochrane Handbook For Systematic Reviews Of Interventions [internet]. Chichester, England: The Cochrane Collaboration; 2011. Available at: www.cochranehandbook.org

36. Yuma-Guerrero P. J., Lawson K. A., Velasquez M. M., von Sternberg K., Maxson T., Garcia N. Screening, brief intervention, and referral for alcohol use in adolescents: a systematic review. Pediatrics 2012; 130: 115-22.

37. Emmen M. J. Effectiveness of opportunistic brief interventions for problem drinking in a general hospital setting: systematic review. BMJ 2004; 328: 318-22.

38. Bertholet N., Daeppen J.-B., Wietlisbach V., Fleming M., Burnand B. Reduction of alcohol consumption by brief alcohol intervention in primary care: systematic review and meta-analysis. Arch Intern Med 2005; 165: 986-95.

39. Moher D., Liberati A., Tetzlaff J., Altman D. G., PRISMA Group. Preferred reporting items for systematic reviews and meta-analyses: the PRISMA statement. Ann Intern Med 2009; 151: 264-9 W64.

40. Blow F. C., Walton M. A., Murray R., Cunningham R. M., Chermack S. T., Barry K. L. et al. Intervention attendance among emergency department patients with alcohol- and drug-use disorders. J Stud Alcohol Drugs 2010; 71: 713-19.
41. Baird J., Longabaugh R., Lee C. S., Nirenberg T. D., Woolard R., Mello M. J. et al. Treatment completion in a brief motivational intervention in the emergency department: the effect of multiple interventions and therapists' behavior. Alcohol Clin Exp Res 2007; 31: 71s-75s.

42. Arean P. A., Ayalon L., Jin C., McCulloch C. E., Linkins K., Chen H. et al. Integrated specialty mental health care among older minorities improves access but not outcomes: results of the PRISM-E study. Int J Geriatr Psychiatry 2008; 23: 1086-92.

43. Oslin D. W., Grantham S., Coakley E., Maxwell J., Miles K., Ware J. et al. PRISM-E: comparison of integrated care and enhanced specialty referral in managing at-risk alcohol use. Psychiatr Serv 2006; 57: 954-8.

44. Young M. M., Stevens A., Porath-Waller A., Pirie T., Garritty C., Skidmore B. et al. Effectiveness of brief interventions as part of the screening, brief intervention and referral to treatment (SBIRT) model for reducing the non-medical use of psychoactive substances: a systematic review protocol. Systematic Reviews 2012; 1: 22.

45. McKellar J., Austin J., Moos R. Building the first step: a review of low-intensity interventions for stepped care. Addict Sci Clin Pract 2012; 7: 26.

46. StataCorp Stata Statistical Software: Release 13. College Station, TX: StataCorp, LP; 2013.

47. Higgins J. P. T., Thompson S. G. Quantifying heterogeneity in a meta-analysis. Stat Med 2002; 21: 1539-58.

48. Sackett D. L., Gent M. Controversy in counting and attributing events in clinical trials. N Engl J Med 1979; 301: 1410-12.

49. Glass J. E., Bucholz K. K. Concordance between self-reports and archival records of physician visits: a case-control study comparing individuals with and without alcohol use disorders in the community. Drug Alcohol Depend 2011; 116: 57-63.

50. Killeen T. K., Brady K. T., Gold P. B., Tyson C., Simpson K. N. Comparison of self-report versus agency records of service utilization in a community sample of individuals with alcohol use disorders. Drug Alcohol Depend 2004; 73: 141-17.

51. Balk E. M., Bonis P. A. L., Moskowitz H., Schmid C. H., Ioannidis J. P. A., Wang C. et al. Correlation of quality measures with estimates of treatment effect in meta-analyses of randomized controlled trials. JAMA 2002; 287: 2973-82.

52. Begg C. Publication bias. In: Cooper H. M., Hedges L. V., editors. The Handbook of Research Synthesis. New York: Russell Sage Foundation; 1994, pp. 399-409.

53. Harbord R. M., Egger M., Sterne J. A. C. A modified test for small-study effects in meta-analyses of controlled trials with binary endpoints. Stat Med 2006; 25: 3443-57.

54. Kuchipudi V., Hobein K., Flickinger A., Iber F. L. Failure of a 2-hour motivational intervention to alter recurrent drinking behavior in alcoholics with gastrointestinal disease. J Stud Alcohol 1990; 51: 356-60.

55. Bischof G., Grothues J. M., Reinhardt S., Meyer C., John U., Rumpf H. J. Evaluation of a telephone-based stepped care intervention for alcohol-related disorders: a randomized controlled trial. Drug Alcohol Depend 2008; 93: 244-51.

56. Copeland L. A., Blow F. C., Barry K. L. Health care utilization by older alcohol-using veterans: effects of a brief intervention to reduce at-risk drinking. Health Educ Behav 2003; 30: 305-21.

57. Wutzke S. E., Conigrave K. M., Saunders J. B., Hall W. D. The long-term effectiveness of brief interventions for unsafe alcohol consumption: a 10-year follow-up. Addiction 2002; 97: $665-75$.

58. Field C. A., Caetano R. The effectiveness of brief intervention among injured patients with alcohol dependence: who 
benefits from brief interventions? Drug Alcohol Depend 2010; 111: $13-20$.

59. Cherpitel C. J., Korcha R. A., Moskalewicz J., Swiatkiewicz G., Ye Y., Bond J. Screening, brief intervention, and referral to treatment (SBIRT): 12-month outcomes of a randomized controlled clinical trial in a Polish emergency department. Alcohol Clin Exp Res 2010; 34: 1922-8.

60. Batel P., Pessione F., Bouvier A. M., Rueff B. Prompting alcoholics to be referred to an alcohol clinic: the effectiveness of a simple letter. Addiction 1995; 90: 811-14

61. Bernstein J., Heeren T., Edward E., Dorfman D., Bliss C., Winter M. et al. A brief motivational interview in a pediatric emergency department, plus 10-day telephone follow-up, increases attempts to quit drinking among youth and young adults who screen positive for problematic drinking. Acad Emerg Med 2010; 17: 890-902.

62. Gentilello L. M., Rivara F. P., Donovan D. M., Jurkovich G. J., Daranciang E., Dunn C. W. et al. Alcohol interventions in a trauma center as a means of reducing the risk of injury recurrence. Ann Surg 1999; 230: 473-80 discussion 480-3.

63. Crawford M. J., Patton R., Touquet R., Drummond C., Byford S., Barrett B. et al. Screening and referral for brief intervention of alcohol-misusing patients in an emergency department: a pragmatic randomised controlled trial. Lancet 2004; 364: 1334-9.

64. Monti P. M., Barnett N. P., Colby S. M., Gwaltney C. J., Spirito A., Rohsenow D. J. et al. Motivational interviewing versus feedback only in emergency care for young adult problem drinking. Addiction 2007; 102: 1234-43.

65. Monti P. M., Colby S. M., Barnett N. P., Spirito A., Rohsenow D. J., Myers M. et al. Brief intervention for harm reduction with alcohol-positive older adolescents in a hospital emergency department. J Consult Clin Psychol 1999; 67: 989-94.

66. Barnett N. P., Murphy J. G., Colby S. M., Monti P. M. Efficacy of counselor vs. computer-delivered intervention with mandated college students. Addict Behav 2007; 32: 2529-48.

67. Center for Substance Abuse Treatment. Brief Interventions and Brief Therapies for Substance Abuse. Rockville, MD: US Substance Abuse and Mental Health Services Administration; 1999.

68. Cherpitel C. J., Bernstein E., Bernstein J., Moskalewicz J., Swiatkiewicz G. Screening, Brief Intervention and Referral to Treatment (SBIRT) in a Polish emergency room: challenges in cultural translation of SBIRT. J Addict Nurs 2009; 20: 127-31.

69. Glass J. E., Kristjansson S. D., Bucholz K. K. Perceived alcohol stigma: factor structure and construct validation. Alcohol Clin Exp Res 2013; 37: E237-46.

70. McCormick K. A., Cochran N. E., Back A. L., Merrill J. O., Williams E. C., Bradley K. A. How primary care providers talk to patients about alcohol: a qualitative study. J Gen Intern Med 2006; 21: 966-72.

71. Amaral-Sabadini M. B., Saitz R., Souza-Formigoni M. L. O. Do attitudes about unhealthy alcohol and other drug (AOD) use impact primary care professionals' readiness to implement AOD-related preventive care? Drug Alcohol Rev 2010; 29: 655-61.

72. McLellan A. T., Meyers K. Contemporary addiction treatment: a review of systems problems for adults and adolescents. Biol Psychiatry 2004; 56: 764-70.

73. National Institute on Alcohol Abuse and Alcoholism. Treatment for Alcohol Problems: Finding and Getting Help. NIH Publication no. 14-7974. Bethesda, MD: Department of Health and Human Services; 2014.

74. Jonas D. E., Amick H. R., Feltner C., Bobashev G., Thomas K., Wines R. et al. Pharmacotherapy for adults with alcohol use disorders in outpatient settings: a systematic review and meta-analysis. JAMA 2014; 311: 1889-900.

75. Oslin D. W., Lynch K. G., Maisto S. A., Lantinga L. J., McKay J. R., Possemato K. et al. A randomized clinical trial of alcohol care management delivered in Department of Veterans Affairs primary care clinics versus specialty addiction treatment. J Gen Intern Med 2014; 29: 162-8.

76. Saitz R., Cheng D. M., Winter M., Kim T. W., Meli S. M., Allensworth-Davies D. et al. Chronic care management for dependence on alcohol and other drugs: the AHEAD randomized trial. JAMA 2013; 310: 1156.

77. Saitz R., Palfai T. P., Cheng D. M., Horton N. J., Dukes K., Kraemer K. L. et al. Some medical inpatients with unhealthy alcohol use may benefit from brief intervention. J Stud Alcohol Drugs 2009; 70: 426-35.

78. Hopewell S., McDonald S., Clarke M., Egger M. Grey literature in meta-analyses of randomized trials of health care interventions. Cochrane Database Syst Rev 2007; 2 MR000010.

79. Scott C. K., Dennis M. L. Results from two randomized clinical trials evaluating the impact of quarterly recovery management checkups with adult chronic substance users. Addiction 2009; 104: 959-71.

\section{Supporting Information}

Additional supporting information may be found in the online version of this article at the publisher's web-site:

Appendix Table S1 Search keywords Appendix Table S2 Reasons for exclusion during full-text review

Appendix Table S3 Components of the intervention and control groups of alcohol brief alcohol interventions assessing post-intervention treatment utilization $(n=13)$ Appendix Table S4 Elements of risk of bias in the included studies 